\title{
Ileal Obstruction, CTCAE
}

National Cancer Institute

\section{Source}

National Cancer Institute. Ileal Obstruction, CT CAE. NCI Thesaurus. Code C57823.

A disorder characterized by blockage of the normal flow of the intestinal contents in the ileum. 